\title{
Delocalized vibrations in classical random chains
}

\author{
Francisco Domínguez-Adame and Enrique Maciá \\ Departamento de Física de Materiales, Facultad de Ciencias Físicas, Universidad Complutense, E-28040 Madrid, Spain \\ Angel Sánchez \\ Escuela Politécnica Superior, Universidad Carlos III de Madrid, Avda. Mediterráneo 20, \\ E-28913 Leganés, Madrid, Spain
}

(Received 17 March 1993)

\begin{abstract}
Normal modes of one-dimensional disordered chains with two couplings, one of them assigned at random to pairs in an otherwise perfect chain, are investigated. We diagonalize the dynamical matrix to find the normal modes and to study their spatial extent. Multifractal analysis is used to discern clearly the localized or delocalized character of vibrations. In constrast to the general viewpoint that all normal modes in one dimensional random chains are localized, we find a set of extended modes close to a critical frequency, whose number increases with the system size and becomes independent of the defect concentration.
\end{abstract}

In his famous paper on vibrations of glasslike disordered chains, ${ }^{1}$ Dean stated that when disorder (in any form) exists in a system the lattice modes are localized. Since Dean's paper was published, it has been claimed that unless the chain is ordered, or unless $\omega=0$, all vibrational modes are localized in one dimension. ${ }^{2}$ Later on, in analogy to previous works concerning related vibrational problems, some authors conjectured that electrons in one-dimensional disordered lattices are also localized. That is, localization of all eigenstates by disorder in onedimensional systems is viewed as an exact statement. ${ }^{3}$ In a series of recent papers, ${ }^{4-7}$ however, $\mathrm{Wu}$, and co-workers have proposed some discrete (tight-binding) models that exhibit metal-insulator transitions in spite of their randomness. These authors have shown that when defects containing a plane of symmetry ${ }^{7}$ are introduced at random in an otherwise ordered chain, $\sqrt{N}$ states ( $N$ being the total number of states) remain unscattered by the disorder and consequently are extended. In addition, Sánchez and Domínguez-Adame ${ }^{8}$ have presented evidence that a large number of states whose localization length is greater than the system size arises in continuous (Kronig-Penney) models, in which $\delta$ functions are regularly spaced and their strength takes two values, one of them in pairs at random (dimer defects). As a consequence, in such random systems electronic transport can take place almost ballistically. In view of these results, it is a natural question to ask whether classical vibrational modes in all random lattices are actually localized. In this paper we attempt to answer this question showing a particular random system that presents a set of delocalized vibrations. We feel that this is a novel result because, whereas electron dynamics is quantum mechanical and tunneling allows for transitions classically forbidden (roughly speaking, tunneling favors delocalization), we are dealing with a purely classical problem -it is well known that energy levels of the vibrating system can be derived by considering the corresponding classical problem. Quantum features of phonons appear in their statistics, not in their dynamics.
A standard way to study the acoustic properties of a lattice is to consider a nearest-neighbor harmonic chain. The equation of motion for the $N$ atoms reads

$$
\begin{array}{r}
m_{n} \frac{d^{2} U_{n}}{d t^{2}}=K_{n} U_{n+1}+K_{n-1} U_{n-1}-\left(K_{n}+K_{n-1}\right) U_{n} \\
n=1, \ldots, N
\end{array}
$$

where $U_{n}$ is the displacement of the $n$th atom from its equilibrium position, $m_{n}$ is the corresponding mass, and $K_{n}$ denotes the strength of the harmonic coupling between atoms. We build our model in the following way: we take all the masses to be the same $m_{n}=m$, and we allow only two values for $K_{n}, K$, and $K^{\prime}$, with the additional constraint that $K^{\prime}$ appears only in pairs. Assuming a time harmonic dependence $U_{n} \propto \exp (i \omega t)$ in Eq. (1), the stationary equation of motion can be cast in the following matrix form:

$$
\begin{aligned}
\left(\begin{array}{c}
U_{n+1} \\
U_{n}
\end{array}\right) & =\left(\begin{array}{cc}
\frac{K_{n}+K_{n-1}-\lambda K}{K_{n}} & -\frac{K_{n-1}}{K_{n}} \\
1 & 0
\end{array}\right)\left(\begin{array}{c}
U_{n} \\
U_{n-1}
\end{array}\right) \\
& \equiv P_{n}(\lambda)\left(\begin{array}{c}
U_{n} \\
U_{n-1}
\end{array}\right)
\end{aligned}
$$

with $\lambda=m \omega^{2} / K$.

Consider now a single defect in which three atoms, placed at sites $l-1, l$, and $l+1$, are coupled by strengths $K^{\prime}$ between them and by strengths $K$ to the surrounding lattice. The transfer matrix across such a defect is simply given by the matrix product $P_{l+1}(\lambda) P_{l}(\lambda) P_{l-1}(\lambda)$. It is a matter of simple algebra to demonstrate that at the particular value $\lambda=2 K^{\prime} / K$ this matrix product equals, appart from a constant phase change without physical relevance, the transfer matrix at any site of the perfect lattice. The meaning of this result is easy to understand: There exists a special frequency $\omega_{c}=\sqrt{2 K^{\prime} / m}$ for which the reflection coefficient at the defect vanishes. Since $\omega_{c}$ must be below the highest frequency of vibrations in the 
perfect lattice $\omega_{\max }=2 \sqrt{K / m}$, the additional condition $K^{\prime}<2 K$ is required. This result is related to that found for electrons in tight-binding models containing symmetrical defects, where the location in the energy band of the unscattered states is determined by the vanishing of the reflection coefficient at a single defect. ${ }^{7}$ It is worth mentioning that the special frequency $\omega_{c}$ is nothing but the frequency of the longitudinal mode of a single atom of mass $m$ attached to two springs of constant $K^{\prime}$ with fixed ends.

Bearing in mind the above result, we now proceed to study the localization properties of vibrating systems when several of such defects are located at random along the chain. For comparison, we have also studied ordinary random chains, that is, without requiring the constraint that $K^{\prime}$ appears in pairs. In addition, we have obtained results both realizationwise and on average. With no loss of generality we set $K^{\prime}=1.5 K$, so the special frequency for which the reflection coefficient at a single defect vanishes is $\omega_{c}=\sqrt{3 K / m}$. For brevity let us denote $\lambda_{c}=m \omega_{c}^{2} / K=3$. Another important parameter in our model is $p$, defined as the ratio between the number of couplings $K^{\prime}$ and the total number of couplings, which we set in the range 0.01 up to 0.8 .

The localized or delocalized character of the vibrations has been elucidated by means of multifractal analysis, a method succesfully used in characterizing electronic wave functions in disordered samples (see Ref. 9 and references therein). The amplitude distribution of normal modes can be characterized by the scaling with the system size of moments associated to the measure defined in the system by us. We use the standard definition of those moments

$$
\mu_{q}(N)=\frac{\sum_{n=1}^{N}\left|U_{n}\right|^{2 q}}{\left(\sum_{n=1}^{N}\left|U_{n}\right|^{2}\right)^{q}} .
$$

Notice that the second moment $\mu_{2}(N)$ coincides with the inverse participation ratio (IPR), as introduced, for instance, in Ref. 10. The multifractal dimension $D_{q}$ is defined via $\mu_{q}(N) \sim N^{-(q-1) D_{q}}$, for $q \neq 1$. For localized vibrations one finds that $D_{q}$ vanishes for all $q$, whereas $D_{q}$ equals unity (the space dimension) for vibrations spreading uniformly. In these two extreme cases trivial multifractal spectra are obtained.

We first describe our studies realizationwise. To find eigenfrequencies and normal modes we directly diagonalize the tridiagonal, symmetrical matrix arising from Eq. (2) with rigid boundaries $\left(U_{0}=U_{N+1}=0\right) .{ }^{11}$ The system size was as large as $N=5000$. We systematically found that vibrations are more delocalized in approaching the critical value $\lambda_{c}$. Figure 1 shows the squared atomic displacements for three different frequencies close to the critical value $\lambda_{c}$ for a system of $N=5000$ and $p=0.2$. A simple inspection of normal modes, however, does not suffice to discuss the localized or delocalized character of vibrations. Usually the IPR works fine to clearly discern localized and extended states. ${ }^{10}$ Delocalized states are expected to present small IPR, of order of $N^{-1}$, while localized states have larger IPR values. A typical situation is presented in Fig. 2(a), where the IPR for a chain with the same parameters as in Fig. 1 is plot-

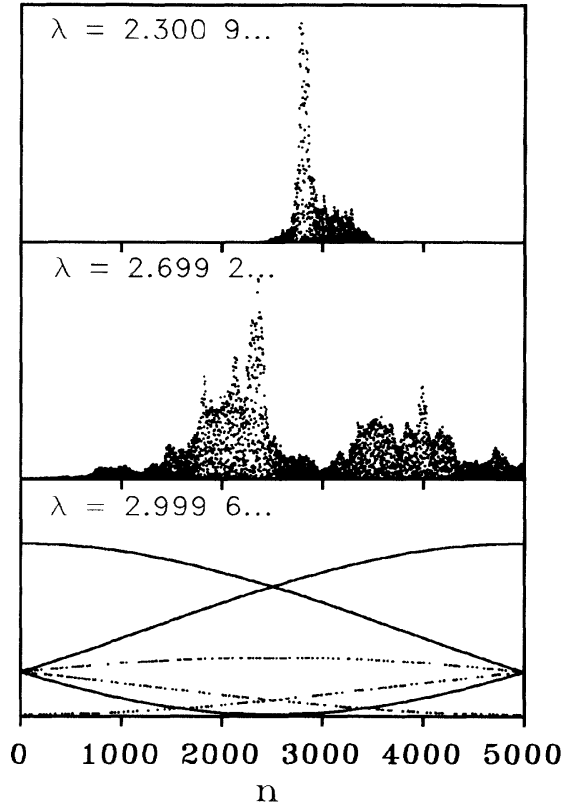

FIG. 1. Squared atomic displacements for three values of the frequency, indicated in each plot, in a system of size $N=5000$ with $p=0.2$. States become more delocalized in approaching the critical value $\lambda_{c}=3$.

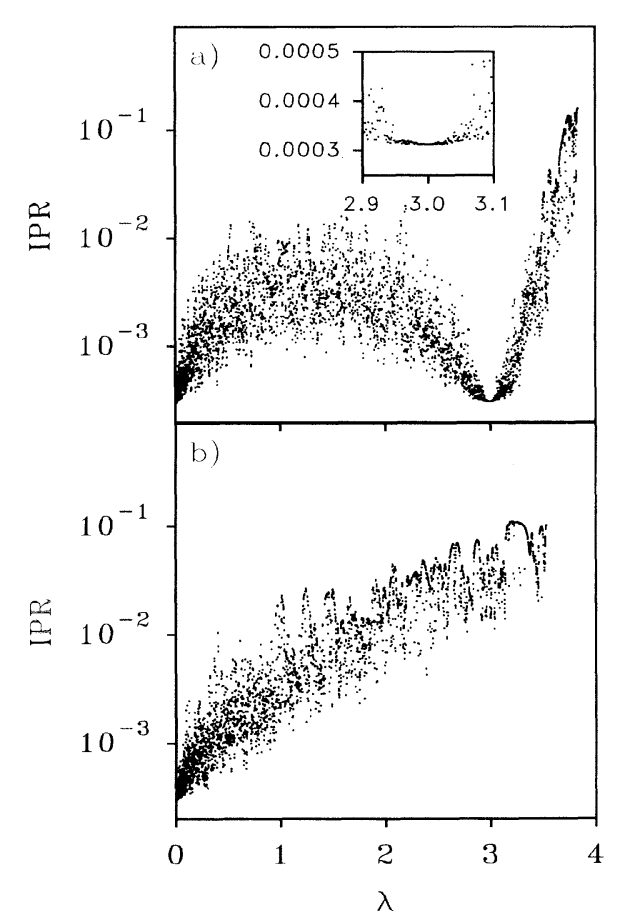

FIG. 2. Inverse participation ratio, obtained by direct diagonalization of the secular matrix, for systems of size $N=5000$ with $p=0.2$ as a function of $\lambda=m \omega^{2} / K$, where $K^{\prime}$ strengths are set (a) at random to pairs and (b) at random. The inset shows the inverse participation ratio close to $\lambda_{c}$ on a linear scale. 
ted vs $\lambda$, in the range $0<\lambda<4$. For comparison the IPR of an ordinary random chain with the same length and the same value of $p$ is shown in Fig. 2(b). One can observe a deep minimum of the IPR around the critical value $\lambda_{c}$, while this minimum is completely absent in ordinary random chains. Interestingly, the values of the IPR at that frequency are similar to those at $\lambda \sim 0$, which so far were believed to be the only ones that could exhibit delocalization properties. Moreover, the inset of Fig. 2(a) shows a plateau close to $\lambda_{c}$ revealing the existence of a set of states with an IPR almost equal to the minimum value. This result indicates that in our model vibrations become extended for frequencies close to $\omega_{c}$. We have estimated that the number of these states is about the square root of the system size, although this result cannot be stated rigorously due to the uncertainty in selecting these states. It is important to mention here that the same results are obtained for larger values of the concentration $p$. In particular, the value of the IPR at $\lambda_{c}$ only depends on the system size but not on $p$. Therefore it seems that the exact number of defects is inmaterial regarding the existence of extended vibrations.

Let us now comment on the average results. We have numerically evaluated the positive integer moments as defined in Eq. (3) with $q=2, \ldots, 6$. Atomic displacements were recursively computed from the transfer matrix equation (2), with the initial conditions $U_{0}=0$ and $U_{1}=1$, and the system size was as large as $N=10^{6}$. When computing averages, they were taken over a number of realizations up to 5000 to exclude errors due to poor statistics. Convergence of moments is reached after very few averages for frequencies close to $\omega_{c}$. This fact is easily understood in view of our previous results: In such a frequency range almost all states are unscattered by defects and consequently no strong fluctuations are expected. Conversely, the number of averages to obtain accurate results increases as one considers more distant frequencies. Our data indicate that 500 averages are enough to investigate the main features of the scaling of moments with system size. Numerical calculations point out that moments scale very accurately as $\mu_{q}(N) \sim N^{-(q-1)}$ for $\lambda=\lambda_{c}$ in all systems we have studied, as illustrated in Fig. 3. Hence the generalized dimension $D_{q}$ at this critical frequency is, within the numerical uncertainty, exactly one, i.e., the space dimension. This means that vibrations spread homogeneously over the whole chain, supporting our claim that those states are completely extended. Close to $\lambda_{c}$ we find that $\mu_{q}(N)$ follows a power law for small $N$ but tends to a constant value for large $N$, as plotted in Fig. 3 for $\lambda=2.90$. The critical size for which deviation from power fit occurs increases in

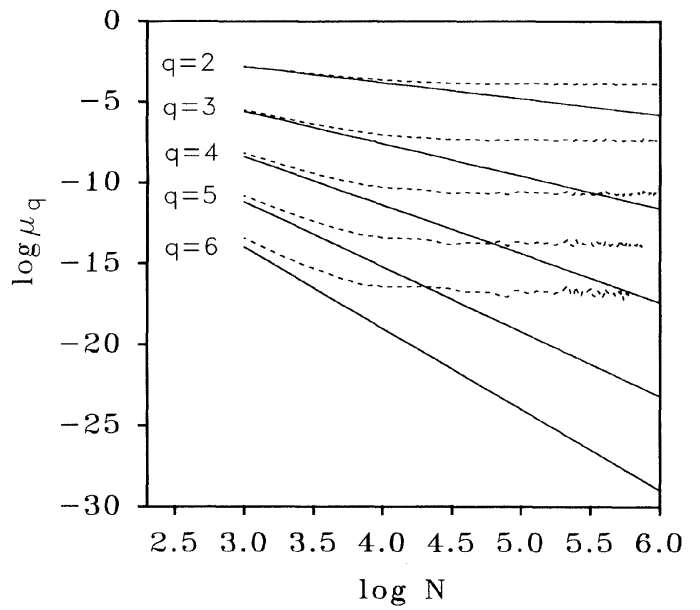

FIG. 3. Scaling of moments with system size for two different frequencies: $\lambda=\lambda_{c}$ (solid line) and $\lambda=2.90$ (dashed line). The concentration of defects is $p=0.2$ in both cases. The logarithms are to base 10 .

approaching $\lambda_{c}$, suggesting that vibrations become more and more delocalized. This is a crucial point because it supports that these are main features of our model irrespective of the particular realization of the disorder.

In summary, we have studied a one-dimensional disordered chain which, in contrast to the generally accepted viewpoint, presents a set of delocalized states close to a critical frequency. The number of such extended states roughly grows with the squared root of the system size. We have shown that correlation in coupling between atoms - foreign springs appear in pairs - leads to a classical resonance effect which allows for such extended vibrations. In our studies, not reported here, we also found that if correlation is introduced in the masses rather that in the couplings - foreign masses appear in pairs - the same resonance effect occurs yielding delocalized vibrations. On the other side, it is clear that the main influence of these states concern transport properties of phonons. Accordingly, when computing the contribution of different modes to the thermal conductivity, as defined in Ref. 12 we have observed a drastic enhancement around the critical frequency. Work currently in progress regarding these topics will be reported elsewhere.

We are indebted to Sergey A. Gredeskul for helpful discussions. We also thank the Comisión Interministerial de Ciencia y Tecnología of Spain for financial support under Project No. MAT90-0544 and the Universidad Carlos III de Madrid for computer facilities.
${ }^{1}$ P. Dean, Proc. Phys. Soc. 84, 727 (1964).

${ }^{2}$ E. H. Lieb and D. C. Mattis, Mathematical Physics in One Dimension (Academic, New York, 1966).

${ }^{3}$ J. M. Ziman, Models of Disorder (Cambridge University Press, London, 1979).
${ }^{4}$ D. H. Dunlap, H.-L. Wu, and P. W. Phillips, Phys. Rev. Lett. 65, 88 (1990).

${ }^{5}$ H.-L. Wu and P. Phillips, Phys. Rev. Lett. 66, 1366 (1991).

${ }^{6}$ P. Phillips and H.-L. Wu, Science 252, 1805 (1991).

${ }^{7}$ H.-L. Wu, W. Goff, and P. Phillips, Phys. Rev. B 45, 1623 
(1992).

${ }^{8}$ A. Sánchez and F. Domínguez-Adame (unpublished).

${ }^{9}$ M. Schreiber and H. Grussbach, Mod. Phys. Lett. B 6, 851 (1992).

10 J. Canisius and J. L. van Hemmen, J. Phys. C 18, 4873 (1985).
${ }^{11}$ W. H. Press, B. P. Flannery, S. A. Teukolsky, and W. T. Vetterling, Numerical Recipes in $C$, 1st ed. (Cambridge University Press, Cambridge, 1989).

${ }^{12}$ H. Matsuda and K. Ishii, Prog. Theor. Phys. Suppl. 45, 56 (1970). 